\title{
Proceedings of the Drug Delivery Australia 2012 symposium
}

\author{
Venue: Monash Institute of Pharmaceutical Sciences, Melbourne, Australia 26-27th \\ November, 2012
}

\author{
Ben J. Boyd • Paul Young
}

Published online: 25 January 2014

(C) Controlled Release Society 2014

Drug Delivery Australia 2012 was the 6th Annual Meeting of the Australian Chapter of the Controlled Release Society. The conference was targeted towards scientists conducting drug delivery research from the engineering, materials science, biology, and clinical fields. Although the primary aim of the conference, and indeed this Special Issue, is to showcase the range of excellent drug delivery research undertaken across Australia, the conference had a strong international feel, with over 160 delegates attending from 14 different countries.

Parallel sessions were held to accommodate the large number of excellent speakers, with themed sessions entitled "Lipid-based drug delivery", "Advanced tools for characterization in drug delivery", "Advanced or just retarded - enhancing and reducing drug uptake", "Macromolecular and vaccine drug delivery", and "Drug delivery to the CNS". The array of articles in this special issue covers the thematic lines of the conference.

The Lipid-based Drug Delivery theme is addressed by two articles in this special issue. The exciting developments towards the translation of the "Lipoceramic" delivery technology, through first-in-man studies on the delivery of ibuprofen, are described by Tan et al., with statistically greater bioavailability being achieved. The critical role of self-assembled structure formation during lipid digestion on drug delivery using lipidbased formulations is also reviewed by Phan et al., challenging the community to link structure formation with biopharmaceutical outcomes to progress the lipid-based drug delivery field.

\footnotetext{
B. J. Boyd $(\bowtie)$

Drug Delivery, Disposition and Dynamics, Monash Institute of Pharmaceutical Sciences, Monash University (Parkville Campus), 381 Royal Pde, Parkville, VIC 3052, Australia

e-mail: Ben.Boyd@monash.edu

\section{P. Young $(\bowtie)$}

Pharmacology, School of Medical Sciences, The Woolcock Institute of Medical Research, The University of Sydney, 431 Glebe Point Rd, Sydney, NSW 2006, Australia

e-mail: paul.young@sydney.edu.au
}

The delivery of vaccines is a hot topic, with Simerska et al. describing novel mannosylated-ovalbumin peptide selfadjuvanting vaccines, which display high stability and low toxicity in vivo. Wilkhu et al., international contributors from Aston University, UK, describe their research into oral delivery of vaccines using non-ionic targeted vesicular carriers. Delivery of peptides across biological barriers, whether intended as vaccines or drugs, is a difficult task to achieve; iontophoresis is often employed to deliver compounds more effectively across the skin, and Krishnan et al. provide a study into the various molecular and application parameters leading to delivery of peptides by iontophoretic methods.

The solid state aspects of oral drug delivery are becoming a critical area of interest as the industry seeks to develop highenergy solid dose forms with enhanced dissolution trajectories and increased drug solubility. The confinement of indomethacin in microcontainers is shown to influence the solid state stability of amorphous drug in the new research presented by Nielsen et al. from the University of Copenhagen.

Controlling local drug delivery is important in reducing systemic side effects and providing the simplest mode of targeted therapy. A mini review on intra-periodontal drug delivery is provided by Chrzanowski et al. Salama et al. provided an interesting study on dual delivery to the oral and pulmonary routes using a novel dual formulation system for orally available and inhalation drugs in combination.

We would like to personally thank the contributors to this special issue for their hard work and excellent submissions, which provide a fabulous assortment of research occurring in drug delivery in Australia and by collaborators and friends abroad. We also thank DDTR for the opportunity to present this research in a great new forum. We look forward to the future participation in the Drug Delivery Australia series.

Conflict of interest statement The authors declare that there is no conflict of interest associated with this publication. 